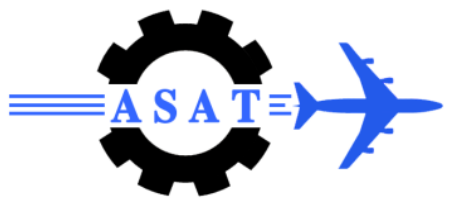

\title{
A Butler Matrix Fed Two-Element Microstrip Antenna Array over EBG Substrate
}

\author{
M.M. Aldemerdash ${ }^{*}$, A.A. Mitkees ${ }^{\dagger}$ and H.A. Almekaty ${ }^{\ddagger}$
}

\begin{abstract}
This paper presents a simulation of two-element microstrip antenna array fed by a Butler matrix over electromagnetic bandgap (EBG) substrate. The proposed structure represents a simple smart antenna system working in the ISM band at $5.8 \mathrm{GHz}$. Small size antenna arrays over high dielectric substrates place the study of mutual coupling problem in an important priority, as it affects the performance of the system. To compensate for mutual coupling effect, mushroom-like EBG structure is used due its winning features. EBG cells are inserted between the two antennas to reduce the mutual coupling effect and to increase the array gain. The performance of the structure with conventional substrate of relative permittivity $\varepsilon_{\mathrm{r}}=10.2$ is compared to that with EBG substrate. The simulation results show that changing the number of EBG columns and rows affects the value of mutual coupling reduction and gain enhancement. Results also indicate that the optimum dimensions of the EBG structure depend on the number of EBG columns and rows. A $26 \mathrm{~dB}$ reduction of mutual coupling is achieved by using 3 columns and 5 rows of EBG structure. Also, a $1.5 \mathrm{~dB}$ improvement of array gain is obtained for the case of 3 columns and 7 rows.
\end{abstract}

Keywords: Smart antennas, microstrip antennas, EBG, Butler matrix, mutual coupling.

\section{Introduction}

Over the last two decades smart antenna technologies, referred to as Multiple Input Single Output (MISO) or Single Input Multiple Output (SIMO), have received much attention. A smart antenna system combines antenna arrays with digital signal processing units in order to improve reception and emission radiation patterns dynamically in response to the signal environment. It can increase channel capacity, extend coverage range, steer multiple beams to track many mobiles, compensate aperture distortion or reduce multipath fading and cochannel interference. A switched beam system is one of the main groups of smart antennas. It forms multiple fixed beams with hightend sensitivity in particular directions [1]. The antenna array of the switched multiple beam system is fed by Butler matrix, due to its advantages such as easy implementation and small path length and number of components [2].

Microstrip antennas are widely used as the array elements of smart antenna systems that are used in small mobile terminals. Applications of microstrip antennas on high dielectric constant substrates are of special interest due to their compact size and conformability with monolithic microwave integrated circuit (MMIC). However, the utilization of a high dielectric

\footnotetext{
*Ph.D. researcher, Mansoura University, mortadadem@gmail.com

'Egyptian Armed Forces, Egypt

Professor, Electronics and Comm. Dept., Mansoura University, h.elmikati@gmail.com
} 
constant substrate has some drawbacks. One of these is the generation of pronounced surface waves that increases the mutual coupling between array elements. The mutual coupling degrades the performance of communication systems which causes the blind angle of a scanning array $[3,4$, and 5].

Several methods have been proposed to reduce the effects of surface waves. One suggested approach is the synthesized substrate that lowers the effective dielectric constant of the substrate either under or around the patch. Another approach is to use a reduced surface wave patch antenna [3]. In recent years, there has been growing interest in utilizing EBG structures in electromagnetic and antenna community to improve the antenna performance. The EBG terminology has been suggested based on photonic band-gap (PBG) phenomena in optics that are realized by periodical structures $[3,5]$. As stated in $[3,5]$ there are diverse forms of EBG structures. The mushroom-like EBG structure has a wining feature of compactness, which is important in the suppression of surface waves propagation, which helps to reduce the mutual coupling in mirostrip antenna arrays. Tan et al provide in [6] significant guidelines in enhancing the performance of the antenna array integrated with mushroom-like EBG. Their results show that not all EBG structures are suitable to improve the performance of the array and in some cases it becomes worse when the EBG is inserted between the microstrip patches. The selection of the size of EBG, the number of columns and spacing between the patches play an important role to improve the performance of the antenna array.

In this paper, a two-element microstrip antenna array fed by a Butler matrix over an EBG substrate is proposed, as shown in Fig. 1-a. The design of mushroom-like EBG is started by calculating its dimensions theoretically using equations given in $[3,5]$. Then, optimizing these dimensions by using Ansoft designer simulator - based on MoM - according to steps stated in [6]. This optimization is done by varying EBG dimensions, number of EBG columns and number of EBG rows, to achieve optimum mutual coupling reduction and gain enhancement.

The paper is organized as follows: In section 2, the design of single microstrip patch antenna with inset feed is presented. Section 3 introduces the design of $2 \times 2$ Butler matrix. Design of the mushroom-like EBG structure is discussed in section 4 . The effect of mutual coupling on the performance of two-element microstrip antenna array is analyzed in section 5. The effect of mutual coupling on the performance of two-element microstrip antenna array fed by a Butler matrix is provided in section 6. Conclusion is reported in section 7.

\section{Design of Microstrip Patch Antenna}

Microstrip patch antennas are suitable for direct integration with microstrip circuits, since it can be etched on the same substrate. Inset feed microstrip line is used here as it is suitable to arrays where the feed network can be printed with the elements. Fig. 1-b. shows the geometry of this antenna designed at the operating frequency $5.8 \mathrm{GHz}$. Ground and patch are made of copper. The dielectric material of the substrate is Rogers RT/duroid 6010/6010LM with dielectric constant $\varepsilon_{\mathrm{r}}=10.2$, dielectric loss tangent tan $\delta=0.0023$ and thickness $\mathrm{h}=1.27 \mathrm{~mm}$. Theoretical design of the microstrip antenna has been done by a MATLAB program using equations given in [1], while the feeding microstrip line has been designed by microwave office (MWO)-TXLINE software. Then design and optimization of the microstrip antenna has been performed by Ansoft Designer with mesh frequency $8 \mathrm{GHz}$.

During design optimization, a good impedance matching is obtained at the operating frequency $5.8 \mathrm{GHz}$ as seen in Figure 2. The optimized antenna parameters are: patch width 
$\mathrm{W}_{\mathrm{p}}=10.9 \mathrm{~mm}$, patch length $\mathrm{L}_{\mathrm{p}}=7.66 \mathrm{~mm}$, feed width $\mathrm{W}_{\mathrm{f}}=1.2 \mathrm{~mm}$, feed length $\mathrm{L}_{\mathrm{f}}=10 \mathrm{~mm}$, gap width $\mathrm{W}_{\mathrm{o}}=1.257 \mathrm{~mm}$ and inset length $\mathrm{y}_{\mathrm{o}}=2.8 \mathrm{~mm}$. Fig. 2 -a shows the input return loss $\mathrm{S}_{11}$, where its minimum value equals $-92.36 \mathrm{~dB}$ at $5.8 \mathrm{GHz}$, and at a reference $\mathrm{S}_{11}=-10 \mathrm{~dB}$, the bandwidth of the antenna is found to be $\mathrm{BW}=76 \mathrm{MHz}(1.31 \%)$. Input impedance is shown in Fig. 2-b, where its value at $5.8 \mathrm{GHz}$ is $50 \Omega$. The radiation pattern at $5.8 \mathrm{GHz}$ is shown in Fig. 3, with antenna gain of $4.47 \mathrm{~dB}$ at broadside.

\section{3. $2 \times 2$ Butler Matrix Design}

The Butler matrix is designed and realized with microstrip technology to feed the antenna array of the proposed structure at frequency $5.8 \mathrm{GHz}$. A $2 \times 2$ butler matrix creates two orthogonal beams in space by processing the signal from the two antenna elements of an equispaced linear array. This Butler matrix is realized using one $3 \mathrm{~dB}, 90^{\circ}$ hybrid coupler. The power applied at a port of the hybrid is evenly distributed between the ports located on the opposite side of it. There is a $90^{\circ}$ phase difference between these two ports; the port closer to the input port is leading in phase by $90^{\circ}$. The port located on the same side as the input port is isolated since there is no power reaching it [7]. With the help of MWO-TXLINE software, the hybrid dimensions have been calculated according to the theoretical design in Fig. 4a. The coupler design has been optimized using Ansoft designer to obtain the desired performance, as shown in Fig. 4b. Simulation has been done under the same conditions used in the design of the antenna. The results when input signal is applied to port (1) while port (4) is matched are given in Fig. 5. As expected the phase difference between port 3 and port 2 is approximately $-90^{\circ}$ and the magnitudes of $S_{21}$ and $S_{31}$ are $-4.56 \mathrm{~dB}$ and $-3.52 \mathrm{~dB}$ respectively (near $3 \mathrm{~dB}$ ). Similar results have been found when input is applied to port (4) and port (1) is matched, but with approximately $+90^{\circ}$ phase difference between port 3 and port 2 .

\section{Design of Mushroom-Like EBG Structure}

The mushroom-like EBG structure was first proposed in [8]. It consists of four parts: a metal ground plane, a dielectric substrate, periodic metal patches on top of the substrate, and vertical vias connecting the patches to the ground plane. This EBG structure exhibits a distinct stopband for surface-wave propagation. The operation mechanism of this EBG structure can be modeled as an LC filter array as illustrated in Fig. 6: the inductor L results from the current flowing through the vias, and the capacitor $\mathrm{C}$ is due to the gap effect between the adjacent patches. For an EBG structure with patch width $\mathrm{W}$, gap width g, substrate thickness $h$ and dielectric constant $\varepsilon_{\mathrm{r}}$, the values of the inductance $\mathrm{L}$ and the capacitance $\mathrm{C}$ are $[3,5$, and 6]:

$$
\begin{gathered}
L=\mu_{o} h \\
C=\frac{W \varepsilon_{o}\left(1+\varepsilon_{r}\right)}{\pi} \cosh ^{-1}\left(\frac{2 W+g}{g}\right)
\end{gathered}
$$

Using $\mathrm{L}$ and $\mathrm{C}$, the central frequency of the band gap and the band width can be calculated by:

$$
\omega=\frac{1}{\sqrt{L C}}
$$




$$
B W=\frac{\Delta \omega}{\omega}=\frac{1}{\eta} \sqrt{\frac{L}{C}}
$$

where $\eta$ is the free space impedance which is $120 \pi$.

These formulations are very simple; however, their results are not very accurate. For example, this model does not consider the via's radius information. There is another accurate but complex model to identify the band gap features, using the theory of transmission lines and periodic circuits [5]. In spite of that, the effect of via radius is not critical here as its value is small compared to the EBG patch dimensions.

There are four methods to characterize the EBG structure: Reflection phase method [5], suspended microstrip [9], driven mode dispersion diagram and eigenmode dispersion diagram [10]. The driven mode dispersion diagram is simple in simulation and has a good agreement with the eigenmode dispersion diagram. For this reason it is used to design the EBG structure either by using Ansoft designer or HFSS simulators. After many trials, it has been found that these methods may not give the required performance of the EBG structure in terms of mutual coupling reduction and antenna array gain enhancement. However, these methods can give an initial estimate for the EBG dimensions which is near to the values given by the simple LC model. Then, the optimum EBG dimensions are parametrically studied and optimized to achieve the required performance.

The EBG design starts with calculating its dimensions using the LC model formulas (1 - 4). The initial dimensions of EBG structure is: $\mathrm{W}=3.6 \mathrm{~mm}$ and $\mathrm{g}=0.2 \mathrm{~mm}$; that gives a band gap of $(5.27-6.13) \mathrm{GHz}$ which includes $5.8 \mathrm{GHz}$. A parametric study is done in section 5 to obtain the exact dimensions of EBG structure starting with the obtained initial values by varying EBG patch width $\mathrm{W}$, spacing between EBG patches $\mathrm{g}$, radius of via $\mathrm{r}$, number of EBG rows and number of EBG columns.

\section{Effect of Mutual Coupling on the Performance of Two Elements Microstrip Antenna Array}

The array is a two identical inset fed microstrip patch antennas separated by a distance $\lambda_{\mathrm{o}} / 2$ (approximately $26 \mathrm{~mm}$ ) where $\lambda_{\mathrm{o}}$ is the design wavelength. This distance is from center to center of the antennas, Fig. 1-a. The two patches are H-coupled as it is suitable for use with beamforming networks like Butler matrix. To study the mutual coupling between the two elements, the array is fed directly without Butler matrix. Hence, the design of the EBG is optimized by minimizing the mutual coupling effect $\left(S_{21}\right)$, so that this design can be used in section 6 .

Simulation of the proposed array is done in two steps. First, it is simulated without using EBG structure. The simulation has been done by Ansoft designer using the same design parameters in the previous sections. The scattering parameters $S_{11}$ and $S_{21}$ at $5.8 \mathrm{GHz}$ are $-19.79 \mathrm{~dB}$ and $-19.57 \mathrm{~dB}$ respectively. The gain of the array is $6.37 \mathrm{~dB}$. Then, simulation has been repeated with the same design conditions after inserting EBG structure columns between the patches. The simulation starts by fixing the number of columns (1,2 and 3) and vary the number of rows (5, 6 and 7). EBG structure dimensions are optimized to give the best mutual coupling reduction and array gain. 
The simulation results are listed in table 1 and are shown in Fig. 7. From the table, it is seen that each case has a different optimized EBG structure dimensions that are near to that calculated in section 4. Increasing the number of columns keeping the same number of rows tends to increase the gain and the mutual coupling reduction. However, there is a limit for the possible number of allocable columns between the antennas, which is 3 columns in our case. It is also noticed that increasing the number of rows keeping the same number of columns, tends to increase the gain but decrease the mutual coupling reduction. The maximum mutual coupling reduction is $26.1 \mathrm{~dB}(133.3 \%)$ and the corresponding enhanced gain is $1.73 \mathrm{~dB}$ (27.15\%), for the case of 3 columns and 5 rows. While using 3 columns and 7 rows gives maximum gain enhancement of $2.3 \mathrm{~dB}(29.2 \%)$ with mutual coupling reduction of $5.72 \mathrm{~dB}$ (36.23\%). It can be seen that the optimum EBG patch width increases with increasing the number of columns and rows, which indicates that each case has a different design.

\section{Effect of Mutual Coupling on the Performance of Two-Element Microstrip Antenna Array Fed by a Butler Matrix}

The aim of this section is to study the impact of mutual coupling reduction on the performance of the proposed structure in terms of the array gain and the main beam angle. The analysis here is based on the optimum design parameters listed in Table 1, and the optimum dimensions of Butler matrix simulated in section 3. This analysis is divided into 3 steps. Firstly, neglecting mutual coupling effect, the radiation pattern of the proposed structure is simply obtained by multiplication of the radiation pattern of the single microstrip antenna (element pattern) by the array factor. The element pattern is obtained in section 2 and shown in Fig. 3. The array factor of the proposed structure is calculated taking into account the phase shifts between the two array inputs from left to right are $-90^{\circ}$ and $90^{\circ}$ [4]. Next, gain and angle of the main beam of the proposed structure are calculated taking into account mutual coupling effect with conventional substrate, where the whole structure is simulated as a single unit. The simulation is done for the two cases of the phase shift stated in section 3 . Finally, the second step is repeated but with employing the EBG substrate.

The calculated and simulated results are shown in Table 2 and illustrated in Fig. 8. It is obvious that the gain of the proposed structure is enhanced with increasing number of EBG structure columns and rows in comparison with conventional structure. The gain is increased by $1.52 \mathrm{~dB}(25.5 \%)$ and $1.45 \mathrm{~dB}(24.2 \%)$ for input phase shifts $-90^{\circ}$ and $90^{\circ}$ respectively, for the case of 3 columns and 7 rows. Moreover, maximum gain achieved by the proposed structure exceeds the ideal case by approximately $0.9 \mathrm{~dB}(13.6 \%)$. It is also noted that the mutual coupling affects the main beam angle by a shift of $\pm\left(0.4^{\circ}-1.6^{\circ}\right)$.

\section{Conclusion}

In this paper, a Butler matrix fed two-element microstrip antenna array over EBG structure is designed and simulated using Ansoft Designer v4. The mushroom-like EBG structure has a winning feature of compactness in this proposed structure. The results verify that there are no fixed optimum dimensions for the EBG structure but every case has its own parameters that give the best mutual coupling reduction and enhanced gain. Furthermore, it is found that mutual coupling should be taken into account, otherwise misleading results are obtained. Mutual coupling decreases the array gain by approximately $9 \%(0.6 \mathrm{~dB})$ w.r.t. the ideal case neglecting mutual coupling. Use of mushroom-like EBG structure reduces the mutual coupling by more than $29.2 \%(5.72 \mathrm{~dB})$ and improves the gain by around $25.5 \%(1.5 \mathrm{~dB})$. The main beam angle is slightly affected mutual coupling by approximately $\pm\left(0.4^{\circ}-1.6^{\circ}\right)$. 


\section{References}

[1] C. Sun, J. Cheng, and T. Ohira, Handbook on Advancements in smart antenna technologies for wireless networks, Information Science reference, 2009.

[2] A. M. El-Tager and M. A. Eleiwa, "Design and implementation of a smart antenna using butler matrix for ISM-band," Progress in Electromagnetics Research Symposium, Beijing, China, March 23-27, 2009, pp. 571-575.

[3] F. yang and Y. Rahmat-Sami, "Microstrip antenna integrated with electromagnetic band-gap (EBG) structures: A low mutual coupling design for array applications," IEEE Trans. Antennas Propagat., vol. 51, No. 10, pp. 2936-2945, Oct. 2003.

[4] C. A. Balanis, Antenna theory: analysis and design, 3rd ed. John Wiley\& Sons, 2005.

[5] F. Yang and Y. Rahmat-Sami, Electromagnetic band gap structures in antenna engineering, Cambridge University Press, 2009.

[6] M. N. Md. Tan, T. A. Rahaman, S. K. A. Rahim, M. T. Ali and M. F. Jamlos, "Antenna array enhancement using mushroom-like electromagnetic band gap (EBG),"Fourth European Conference on Antennas and Propagation. EuCAP, Barcelona, Spain, 12-16 April 2010. pp. 1-5.

[7] Wu, Liangjum, "Planar multi-beam antenna for W-LAN," Duisburg, November 2007.

[8] D. Sievenpiper, L. Zhang, R. F. J. Broas, N. G. Alexopolus, and E. Yablonovitch, "High-impedance electromagnetic surfaces with a forbidden frequency band," IEEE Trans. Microwave Theory Tech., vol. 47, pp. 2059-2074, November 1999.

[9] L. Yang, M. Fan, F. Chen, J. She, and Z. Feng, "A novel compact electromagneticbandgap (EBG) structure and its applications for microwave circuits," IEEE Trans. Microwave Theory Tech., vol. 53, No. 1, pp. 183-190, January 2005.

[10] Left-handed Metamaterial design guide using Ansoft Designer and HFSS, www.ansoft.com.

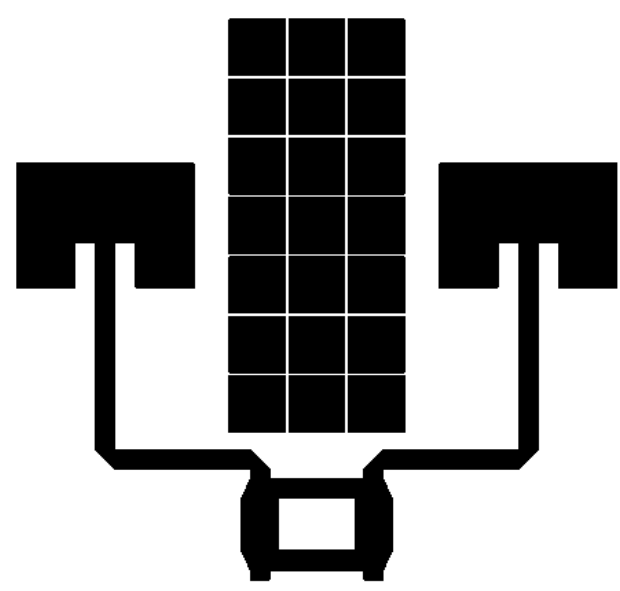

(a)

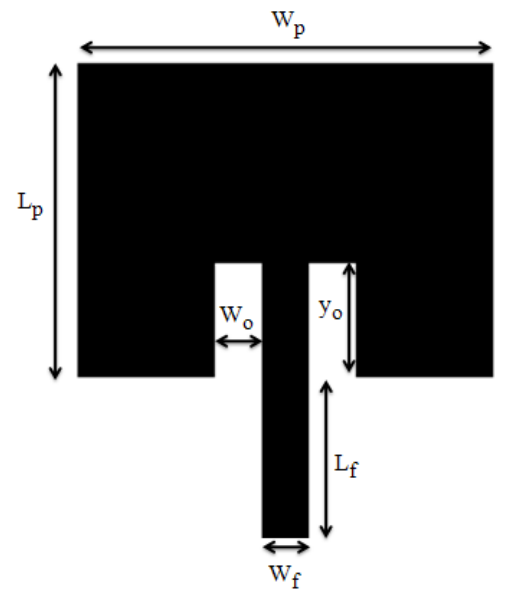

(b)

Fig. 1 (a) $2 \times 2$ fixed beamformer over an EBG substrate. (b) Microstrip patch antenna with inset feed. 


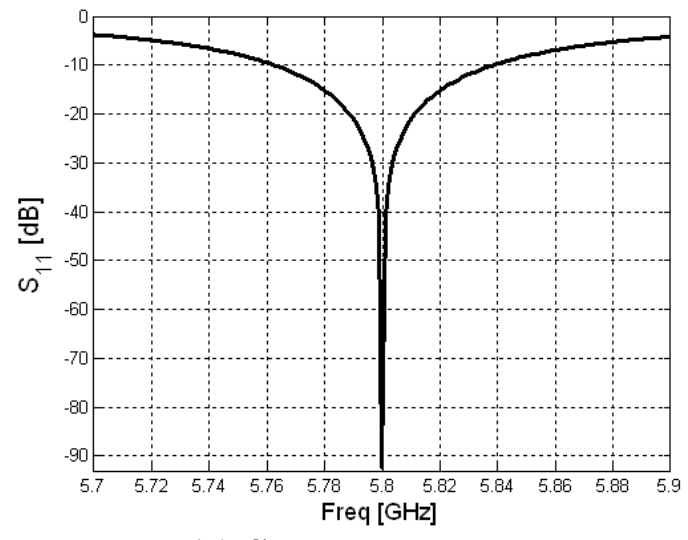

(a) $S_{11}$ parameter.

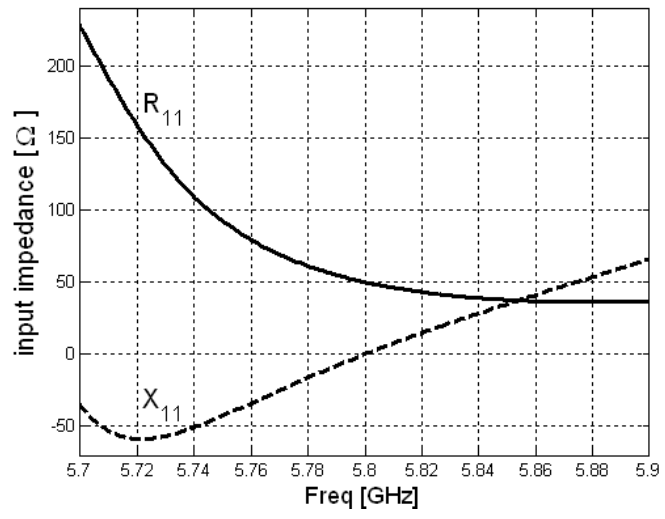

(b) Input impedance.

Fig. 2 Characteristics of one-microstrip antenna with inset feed.

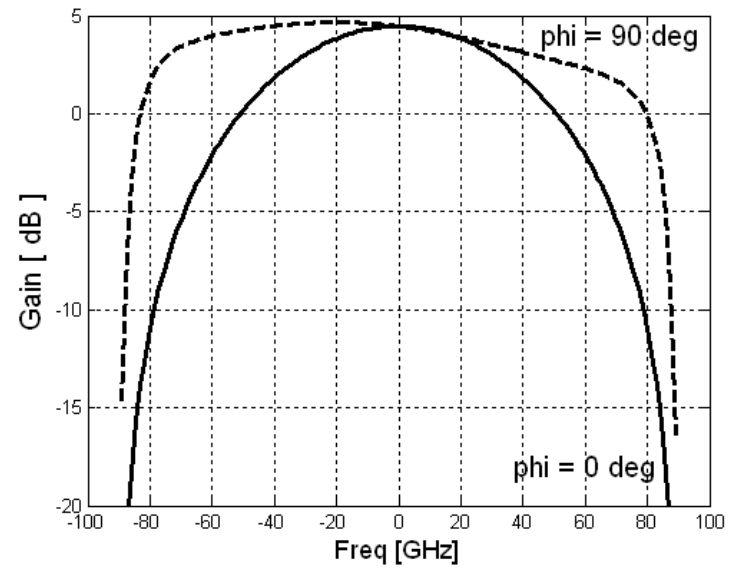

(a) Rectangular

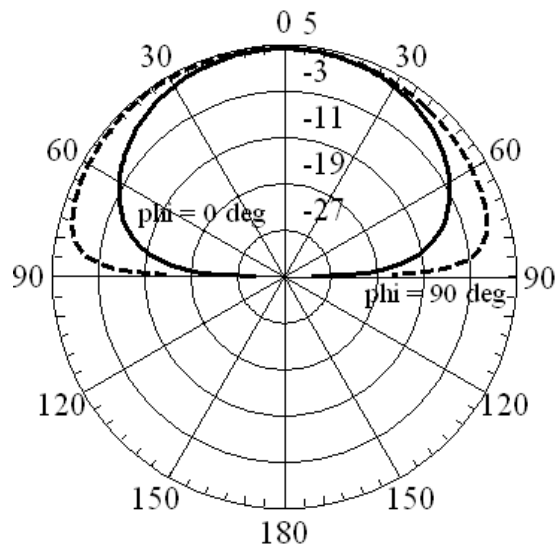

(b) polar

Fig. 3 Radiation pattern of one-microstrip antenna with inset feed in $\mathrm{dB}$ at $5.8 \mathrm{GHz}$. 


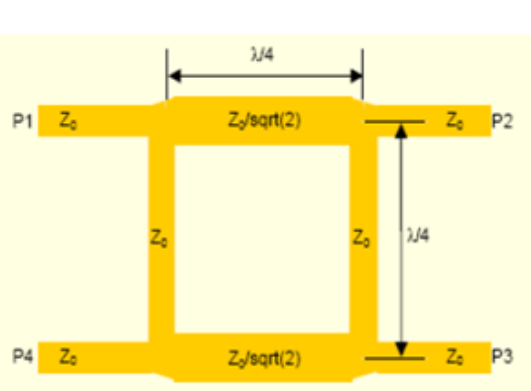

(a)

Theoretical parameters for design.

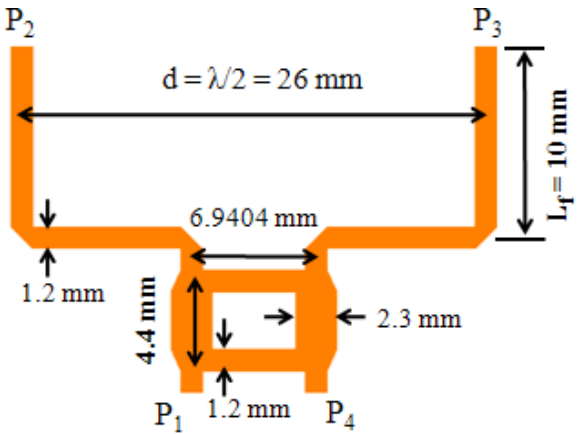

(b)

Parameters Optimized using Ansoft designer v4.

Fig. $43 \mathrm{~dB} \mathrm{90}{ }^{\circ}$ Hybrid Coupler.

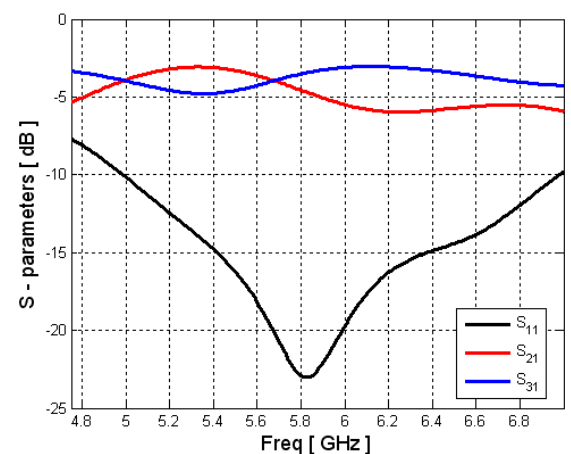

(a) S-parameters.

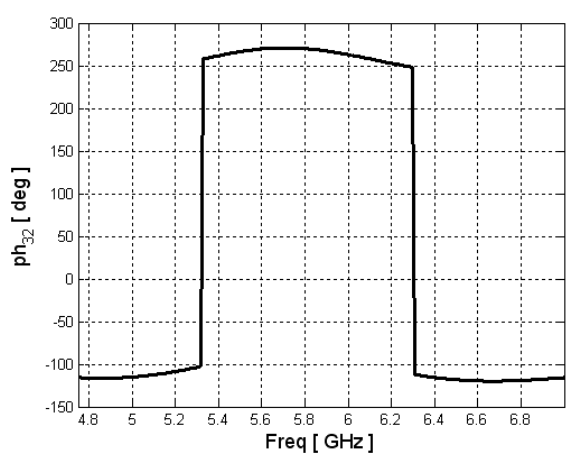

(b) Output phase shift.

Fig. $52 \times 2$ Butler matrix characteristics.

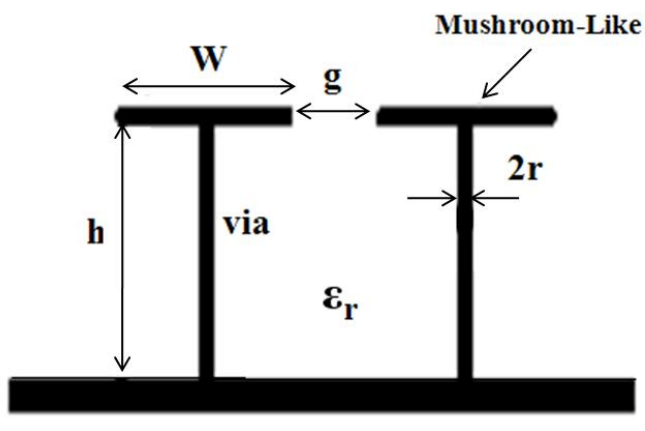

(a)

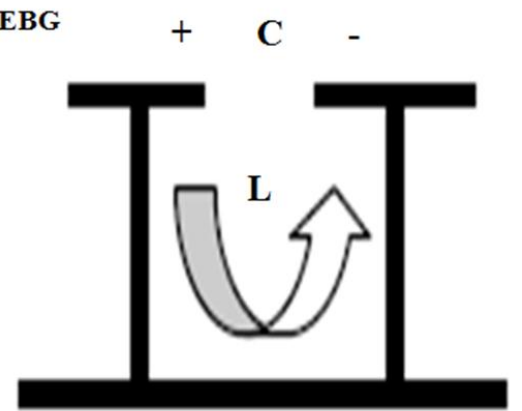

(b)

Fig. 6 (a) Mushroom-like EBG structure parameters

(b) Lumped LC model. 
Table 1 Influence of mushroom-like EBG structure on the performance of two-microstrip antennas array.

\begin{tabular}{|c|c|c|c|c|c|c|}
\hline \multirow{2}{*}{$\begin{array}{l}\text { Number of rows } \\
\text { and columns }\end{array}$} & \multirow{2}{*}{$\begin{array}{c}S_{11} \\
{[\mathrm{~dB}]}\end{array}$} & \multirow{2}{*}{$\begin{array}{c}S_{21} \\
{[\mathrm{~dB}]}\end{array}$} & \multirow{2}{*}{$\begin{array}{l}\text { Gain } \\
{[\mathrm{dB}]}\end{array}$} & \multicolumn{3}{|c|}{ EBG structure parameters } \\
\hline & & & & $\begin{array}{c}w_{e} \\
{[\mathrm{~mm}]}\end{array}$ & $\begin{array}{c}g_{e} \\
{[\mathrm{~mm}]}\end{array}$ & $\begin{array}{c}r_{v} \\
{[\mathrm{~mm}]}\end{array}$ \\
\hline Without EBG & -19.7917 & -19.5757 & 6.3661 & - & - & - \\
\hline \multicolumn{7}{|l|}{$\begin{array}{c}\text { With EBG } \\
1-1 \text { column }\end{array}$} \\
\hline 5 rows & -20.4515 & -25.1891 & 7.4534 & 3.16 & 0.2 & 0.117 \\
\hline 6 rows & -19.9259 & -24.4560 & 7.7082 & 3.20 & 0.2 & 0.115 \\
\hline 7 rows & -17.3717 & -22.6126 & 7.7303 & 3.23 & 0.2 & 0.115 \\
\hline \multicolumn{7}{|l|}{$2-2$ columns } \\
\hline 5 rows & -18.1338 & -29.8289 & 7.8596 & 3.32 & 0.2 & 0.117 \\
\hline 6 rows & -17.2037 & -25.7812 & 8.1002 & 3.36 & 0.2 & 0.115 \\
\hline 7 rows & -17.4862 & -23.9285 & 8.1329 & 3.38 & 0.2 & 0.115 \\
\hline \multicolumn{7}{|l|}{$3-3$ columns } \\
\hline 5 rows & -14.2403 & -45.6486 & 8.0925 & 3.42 & 0.2 & 0.120 \\
\hline 6 rows & -14.8262 & -30.3964 & 8.6030 & 3.44 & 0.2 & 0.114 \\
\hline 7 rows & -14.8112 & -25.2926 & 8.6700 & 3.46 & 0.2 & 0.114 \\
\hline
\end{tabular}

Table 2 Influence of mushroom-like EBG structure on the performance of $2 \times 2$ fixed beam former.

\begin{tabular}{|c|c|c|c|c|}
\hline \multirow[b]{2}{*}{ Number of rows and columns } & \multicolumn{2}{|c|}{$\Delta \beta \approx-90^{\circ}$} & \multicolumn{2}{|c|}{$\beta \approx 90^{\circ}$} \\
\hline & $\begin{array}{c}\text { angle } \\
\text { [ degree ] }\end{array}$ & $\begin{array}{c}\text { Gain } \\
{[\mathrm{dB}]}\end{array}$ & $\begin{array}{c}\text { angle } \\
\text { [ degree ] }\end{array}$ & $\begin{array}{l}\text { Gain } \\
{[\mathrm{dB}]}\end{array}$ \\
\hline Mutual coupling neglected & 19.2 & 6.5658 & -19.2 & 6.5626 \\
\hline $\begin{array}{l}\text { Mutual coupling considered without } \\
\text { EBG }\end{array}$ & 24.4 & 5.9092 & -22.8 & 6.0302 \\
\hline \multicolumn{5}{|l|}{$\begin{array}{l}\text { Mutual coupling considered with EBG: } \\
\qquad 1-1 \text { column }\end{array}$} \\
\hline 5 rows & 24.0 & 6.3719 & -23.1 & 6.4826 \\
\hline 6 rows & 23.5 & 6.6180 & -23.8 & 6.6297 \\
\hline 7 rows & 23.6 & 6.7076 & -23.2 & 6.7040 \\
\hline \multicolumn{5}{|l|}{$2-2$ columns } \\
\hline 5 rows & 23.2 & 6.8072 & -24.4 & 6.6058 \\
\hline 6 rows & 23.9 & 6.8826 & -23.6 & 6.8831 \\
\hline 7 rows & 23.5 & 6.9884 & -23.2 & 6.9892 \\
\hline \multicolumn{5}{|l|}{$3-3$ columns } \\
\hline 5 rows & 24.4 & 7.0066 & -24.0 & 7.0058 \\
\hline 6 rows & 24.8 & 7.1643 & -23.6 & 7.2570 \\
\hline 7 rows & 24.1 & 7.4205 & -23.5 & 7.4662 \\
\hline
\end{tabular}







(a) S-parameters

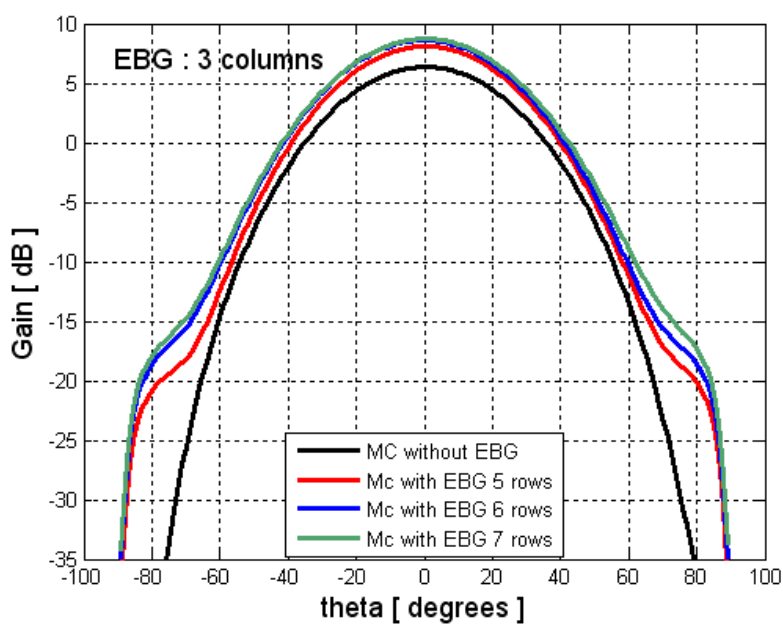

(b) Radiation pattern

Fig. 7 Mutual coupling reduction of a two microstrip antennas array as a result of mushroom-like EBG structure. 

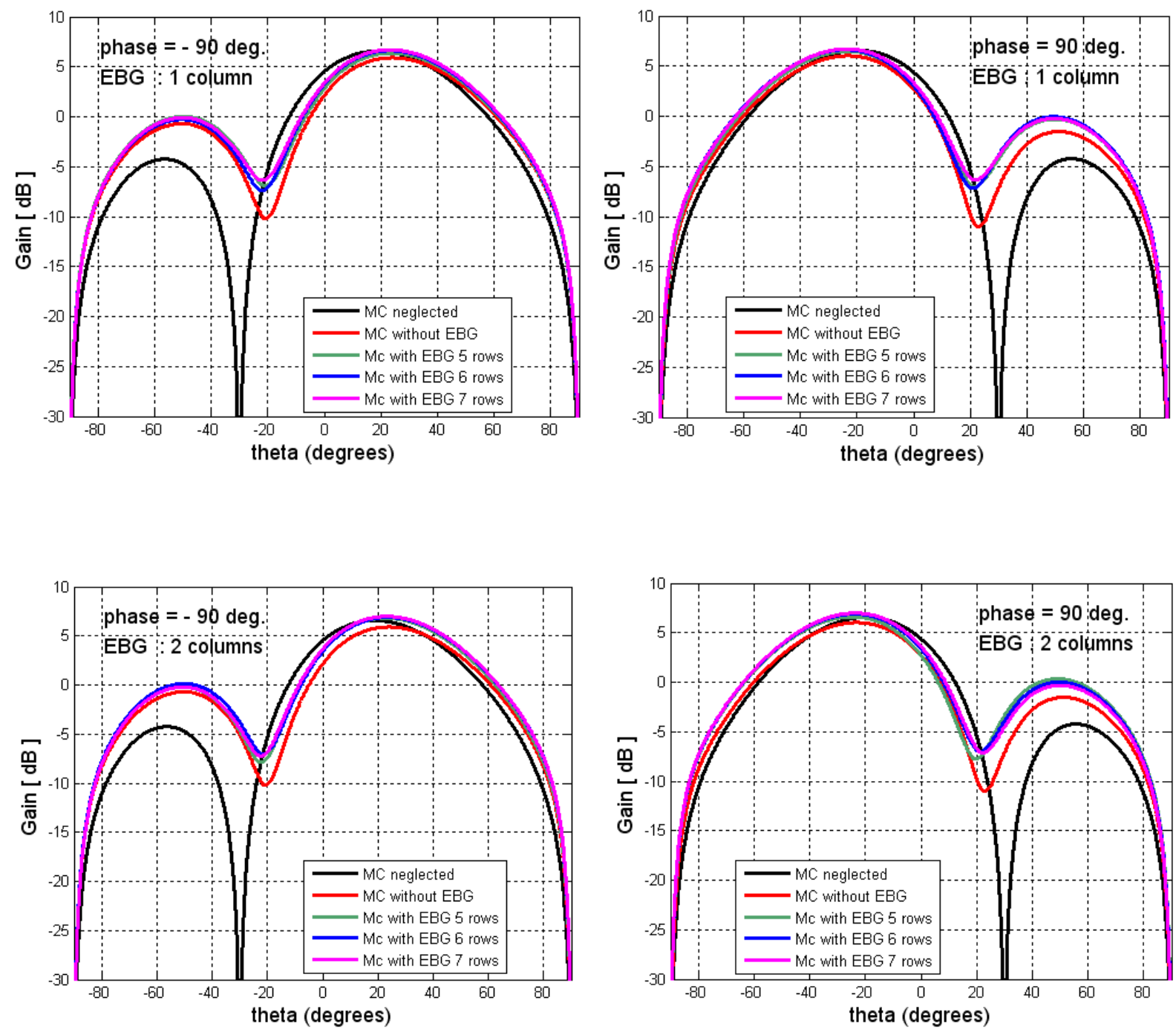



(a) $\Delta \beta \approx-90^{\circ}$

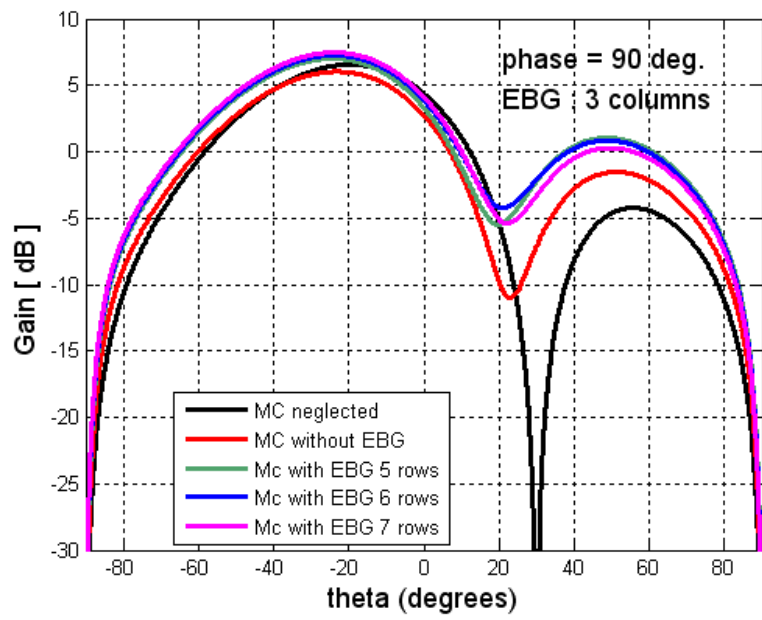

(b) $\Delta \beta \approx 90^{\circ}$

Fig. 8 Effect of mutual coupling reduction on Gain enhancement and main beam angle of a two-microstrip antenna array fed be a Butler matrix. 